\title{
Pavlovian contingencies and anticipatory contrast
}

\author{
BEN A. WILLIAMS \\ University of California, San Diego, La Jolla, California
}

\begin{abstract}
Pigeons were trained on a four-component multiple schedule in which two target components with identical reinforcement schedules were followed by other components with either higher or lower reinforcement rates. The Pavlovian signal properties of the target-component stimuli were varied by changes in their duration relative to the following components, and by whether the two following components were cued by the same or different stimuli. When different stimuli occurred in the following components, response rates were higher in the target component preceding the following component with the lower reinforcement rate, and these contrast effects were larger with shorter relative durations. But with nondifferential stimuli in the following components, contrast consistently occurred only with the longer durations of the target components. Moreover, several subjects with the shorter duration target stimuli had higher response rates in the target followed by the richer schedule-that is, Pavlovian conditioning occurred to the target stimuli. This interaction suggests that the processes underlying anticipatory contrast and Pavlovian conditioning are in opposition, and that the Pavlovian effect can dominate if the signaling properties of the target components are sufficiently enhanced.
\end{abstract}

Numerous experiments have demonstrated that a major portion of behavioral contrast is due to the following schedule (Williams, 1976, 1979, 1981; Williams \& Wixted, 1986; Wilton \& Gay, 1969). The typical procedure used in such experiments has been for two target components, associated with different stimuli but similar reinforcement schedules, to be followed by components with differential reinforcement schedules. Thus, one target component leads to a richer reinforcement schedule; the second leads to a leaner reinforcement schedule. "Anticipatory contrast" has been the result, as response rates in the target components are inversely related to the following rate of reinforcement.

The occurrence of anticipatory contrast is paradoxical when viewed from the perspective of Pavlovian conditioning. In order for differential behavior to occur during the target components, their keylight signals must "predict" the following conditions of reinforcement. But such a predictive relation has been used in other procedures to generate autoshaping (Brown \& Jenkins, 1968). That is, the keylight that predicts the following higher rate of reinforcement should elicit higher response rates, which should result in the opposite of the contrast effects actually obtained.

A major difference between the Pavlovian contingencies embedded in the anticipatory-contrast procedure and the usual autoshaping procedure is that the target stimuli in the former procedure do not lead directly to food, but lead

This research was supported by PHS Grant MH 42797 to the University of California, San Diego. Requests for reprints may be sent to the author, Department of Psychology, C-009, University of California, San Diego, La Jolla, CA 92093. instead only to different following components correlated with differential food presentation. But this cannot be the critical difference because an autoshaping effect has in fact been demonstrated by Brown, Hemmes, Coleman, Hassin, and Goldhammer (1982), using a procedure quite similar to that for anticipatory contrast. In their study, different colored keylights were presented for 8-sec durations, and were then followed by $30 \mathrm{sec}$ of a white keylight. No reinforcement was available during the colors, whereas reinforcement was sometimes available during the white light on a variable-interval (VI) schedule. The major variable was whether the food availability during the white light was correlated with the preceding colors. When only one color was followed by the VI schedule while the other was followed by extinction, high response rates occurred to the former, thus demonstrating autoshaping. When both colors were followed by the VI schedule, little responding occurred to either. Brown et al. proposed that elicited responding to the keylight stimuli was controlled by the relative delay to reinforcement: the stimulus that led to the relatively shorter time to reinforcement generated responding; the other stimulus did not. Thus, autoshaping does not depend upon the keylight stimuli predicting only immediate food presentations, but also occurs when they differentially predict food presentations that occur even after an intervening delay.

The issue raised is why a signal leading to a higher reinforcement rate produces excitation in the autoshaping procedure but response suppression in the contrast procedure. In the present study, this issue was addressed by systematically examining the role of two of the procedural differences between the previous contrast procedures (e.g., Williams, 1979) and the autoshaping procedures 
used by Brown et al. (1982). In the latter procedure, the duration of the target stimuli was much shorter than the period of the following schedule, which should be expected to amplify the "predictiveness" of the target stimuli for the following reinforcement conditions. That is, previous research on autoshaping (Gibbon, Baldock, Locurto, Gold, \& Terrace, 1977) has shown that the speed of conditioning is controlled by the average delay to reinforcement signaled by the conditional stimulus (CS) relative to the average interreinforcement interval; faster conditioning thus occurs with shorter CS durations.

The autoshaping procedure of Brown et al. (1982) also differed from the contrast procedure in that the different following schedules (VI or extinction) were cued by the same white keylight; the only basis for predicting the schedule was the preceding target stimulus. In comparison, the different following schedules in the contrast procedure have been cued by their own differential stimuli, which should serve to overshadow any learning of the association between the target stimuli and the different following schedules. Thus, the absence of differential stimuli in the following components also should create a greater predictiveness of the following schedules by the target stimuli. Both procedural differences between Brown et al.'s study and the contrast studies are in favor of greater "Pavlovian predictiveness" for the target stimuli, which suggests that when the Pavlovian relation between the target stimuli and the following schedules is strengthened, autoshaping, rather than contrast, will be the result.

In the present study, pigeons were presented with a fourcomponent multiple schedule like that used in previous contrast research. A factorial design was used in which under some conditions the target stimuli $(10 \mathrm{sec})$ were shorter than the stimuli correlated with the following schedules $(30 \mathrm{sec}$ ), while under other conditions, they were of the same duration (all $30 \mathrm{sec}$ ). The second factor was whether the stimuli for the different following schedules were different, or always the same. By isolating the effects of these variables, the circumstances producing autoshaping versus contrast should be clarified.

\section{METHOD}

\section{Subjects}

Sixteen White Carneaux pigeons were maintained at $80 \%$ of their free-feeding weights by additional feeding, when necessary, after the termination of the experimental sessions. All had experience in an undergraduate laboratory in which they had been trained under various simple-reinforcement schedules to peck a white keylight.

\footnotetext{
Apparatus

Four identical three-key operant chambers were used. Their internal dimensions were $36 \mathrm{~cm}$ wide $\times 32 \mathrm{~cm}$ long $\times 35 \mathrm{~cm}$ high. All walls were opaque gray plastic except the front, which was sheet aluminum. Mounted on the front sheet were three translucent 2.5cm-diam response keys mounted $26 \mathrm{~cm}$ above the floor and $7.25 \mathrm{~cm}$ apart, center to center. Each key required a force of approximately $0.15 \mathrm{~N}$ to operate and could be transilluminated from the rear by standard IEE 28-V 12-stimulus in-line projectors. A 28-V 1-W miniature lamp was located $8.75 \mathrm{~cm}$ above each response key, and
}

the right-most of these lamps provided general chamber illumination. Directly below the center key and $9.5 \mathrm{~cm}$ above the floor was a $5.7 \times 5 \mathrm{~cm}$ opening that provided access to a solenoid-operated grain hopper. When activated, the hopper was illuminated from above by a $28-\mathrm{V} 1-\mathrm{W}$ miniature lamp. Reinforcement consisted of 3-sec access to milo during which all keylights and houselights were dark. A 5-cm speaker was mounted above the center of the ceiling and provided continuous white noise throughout the experimental sessions. The experimental events in each chamber were controlled by an Apple IIe computer located in an adjacent room.

\section{Procedure}

Because all subjects had prior keypecking experience, they were begun immediately on the four-component multiple schedule in which two of the schedules were VI 2 min (the target components), whereas a VI 30-sec and a VI 6-min schedule (the following schedules) were the remaining two. The two target components, for which the keylight stimuli were red and blue, were presented on the left key. The two following components were presented on the right key. During the nondifferential conditions, the stimulus for both following components was always a vertical white line. During differential conditions, the stimulus associated with the VI 30-sec schedule was a vertical white line, whereas the stimulus associated with the VI 6-min schedule was a small white circle. When any of these stimuli were illuminated, the remaining keys in the chamber were dark and pecks to the dark keys had no scheduled effect.

The duration of both following components was always $30 \mathrm{sec}$. The duration of the two target components, which was held the same within a condition, was either 10 or $30 \mathrm{sec}$. Thus, four separate experimental conditions were defined by all combinations of targetcomponent duration and whether the following components were differentially or nondifferentially signaled. Within a given condition, a particular target component was always followed by the same following component. Within a session, these pairs of components were randomly interspersed. Total session duration was $56 \mathrm{~min}$ for all conditions.

Four subjects were assigned in Phase 1 to each of the four experimental conditions. The schedule following the red target component was initially VI $30 \mathrm{sec}$; that following the blue target component was VI $6 \mathrm{~min}$. After 30 sessions of training, the relation between the target components and the following components was reversed, as shown in Table 1 , and 30 additional sessions were presented. At the start of Phase 2, the relation between the targets and the following components remained the same, and the subjects were randomly reassigned to one of the remaining conditions. After 30 sessions, the relation between the targets and the following schedules was reversed. As shown in Table 1, training consisted of four separate phases, each of which included an initial phase of training and then a reversal. Thus, each phase continued for a total of 60 sessions. By the end of training, all 16 subjects had been exposed to all four experimental conditions, and for each condition both target stimuli had been followed by the two different following schedules.

Table 1

Design of Experiment

\begin{tabular}{cllc}
\hline & & \multicolumn{2}{c}{ Schedule Following } \\
\cline { 3 - 4 } Phase & Condition & Red & Blue \\
\hline 1 & Initial & VI $30 \mathrm{sec}$ & VI $6 \mathrm{~min}$ \\
& Reversal & VI $6 \mathrm{~min}$ & VI $30 \mathrm{sec}$ \\
2 & Initial & VI $6 \mathrm{~min}$ & VI $30 \mathrm{sec}$ \\
& Reversal & VI $30 \mathrm{sec}$ & VI $6 \mathrm{~min}$ \\
3 & Initial & VI $30 \mathrm{sec}$ & VI $6 \mathrm{~min}$ \\
& Reversal & VI $6 \mathrm{~min}$ & VI $30 \mathrm{sec}$ \\
4 & Initial & VI $6 \mathrm{~min}$ & VI $30 \mathrm{sec}$ \\
& Reversal & VI $30 \mathrm{sec}$ & VI $6 \mathrm{~min}$ \\
\hline
\end{tabular}




\section{RESULTS}

The mean response rates in the two target components are shown in Figure 1 for each of the four conditions. The means represent both the initial and the reversal phases of each condition, which were used to cancel out the effects of any stimulus preferences. For three of the four conditions, response rates in the target followed by the VI 6-min component were higher than those in the target followed by the VI 30-sec component, hence demonstrating anticipatory contrast. The effects of the signal and component-duration variables appear not to be additive, but interactive. The biggest difference between the target components occurred with the signal condition with short components, whereas the one condition without a differential effect was that in which the short components were presented without differential signals. The signaling variable exerted little effect for the conditions in which 30 -sec target components were presented.

The differences during the initial sessions of exposure to each condition are relatively meaningless because they are in large part due to the condition that immediately preceded them (see Table 1), and these were variable for different subjects because of the random orders of presen-
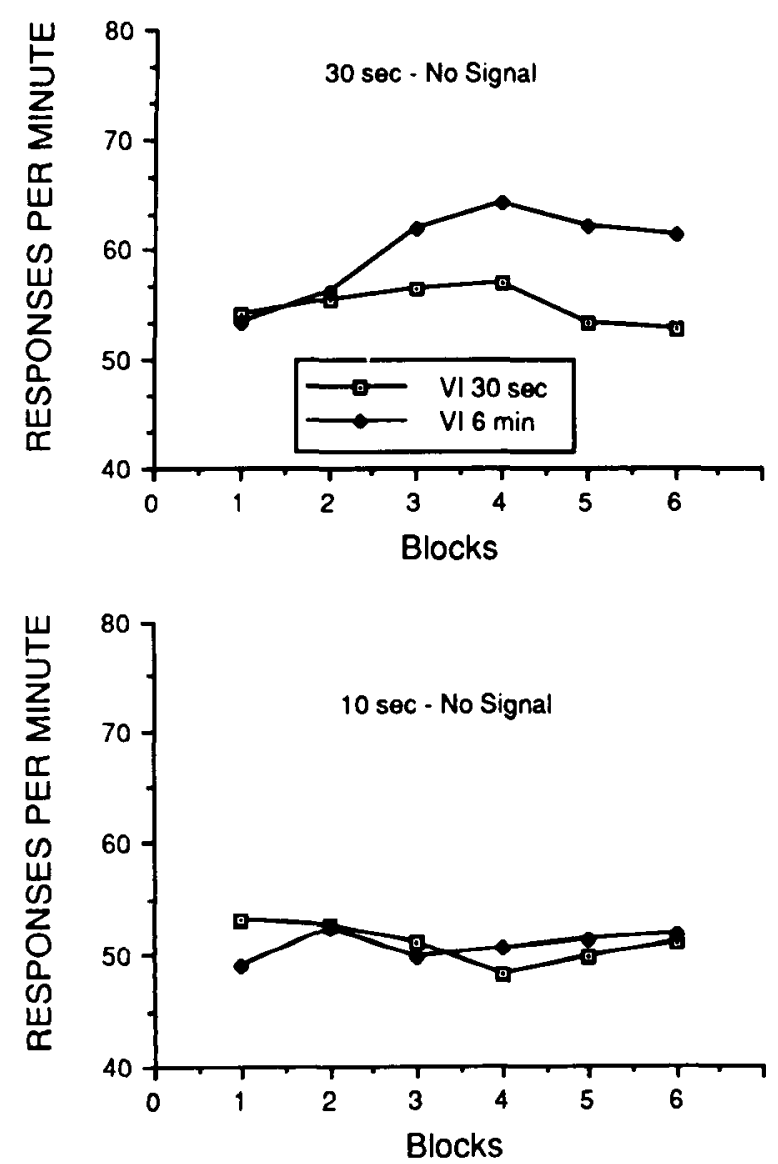

tation. Nevertheless, the two conditions in which the different following components were differentially signaled (right panels of Figure 1) quickly developed differential responding during the first 10 sessions of training, whereas those without differential signals did not. This is not surprising, given that differential performance in the two following components developed only slowly when they were correlated with the same stimulus (see Figure 2, to be discussed below).

The major results of interest are those for the final sessions of each condition after the behavior had stabilized. Figure 1 shows that behavior was generally stable during the last 15 sessions of training, so a four-way analysis of variance (ANOVA) was conducted on those data (duration $\times$ signal $\times$ following schedule $\times$ blocks), using a .05 confidence level. The only main effect that was significant was that of the following schedule $[F(1,15)=$ 9.33]. The interaction of signal $\times$ following schedule was also significant $[F(1,15)=9.43]$, and an analysis of its simple effects demonstrated that the effect of the following schedule was significant only when differential signals occurred in the following components.

The triple interaction of duration $\times$ signal $\times$ following schedule was also significant $[F(1,15)=7.96]$. Be-
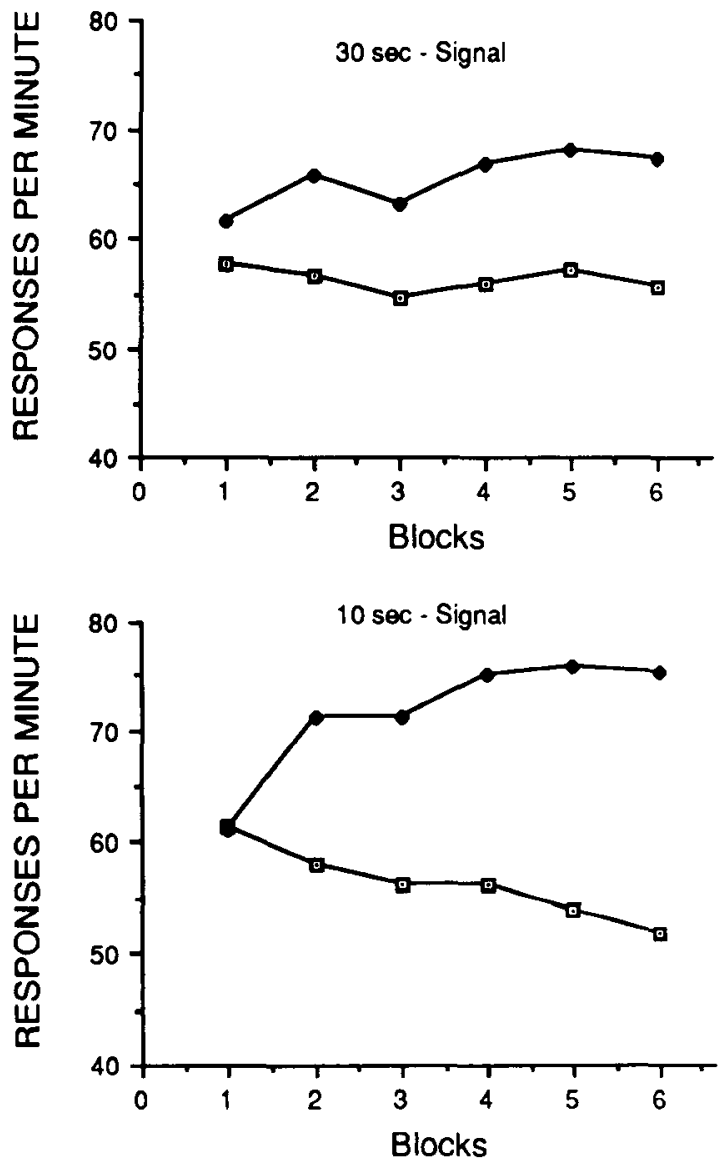

Figure 1. Average response rates across blocks of five sessions for each experimental condition. Separate functions are shown for the target component followed by the VI 30-sec schedule and for the target component followed by the VI 6-min schedule. 
cause of this interaction, separate three-way ANOVAs were conducted on the comparison of the two $30-\mathrm{sec}$ component conditions and of the two 10-sec component conditions. Analysis of the long-component conditions revealed no significant differences, except for that of the following schedule $[F(1,15)=16.10]$. Analysis of the short-component conditions produced a significant difference for the following schedule $[F(1,15)=6.24]$ and for the interaction between signal and following schedule $[F(1,15)=9.13]$. Analysis of simple effects for the interaction term revealed that the effect of the following schedule was significant for the signaled condition $[F(1,15)=8.47]$, but not for the no-signal condition $(F<1.0)$.

The discrimination performance during the two following components is shown in Figure 2. The ordinate is the percentage of the total responses during the VI $30-\mathrm{sec}$ and VI 6-min components that occurred during the VI 30-sec schedule (in other words, the relative rate of responding to the VI 30-sec schedule). Not surprisingly, when the different following schedules were differentially signaled, differential performance quickly developed and was near its asymptotic level during the third block of sessions (Sessions 11-15). There was no difference in this performance as a function of the duration of the target components. When differential signals were not presented during the following components, differential performance was slower to develop and reached a lower asymptote. In addition, discrimination performance was slightly better when the target components were of 30-sec durations.

The results shown in Figure 2 were analyzed with a three-factor ANOVA (duration $\times$ signal $\times$ blocks). The main effects of signal $[F(1,5)=179.9]$ and blocks $[F(5,75)=99.5]$ were significant, as were the interactions of duration $\times$ signal $[F(1,15)=11.39]$ and signal $\times$ blocks $[F(5,75)=2.40]$. Analysis of the simple effects for the interaction of duration $\times$ signal revealed that there was a significant effect of duration for the no-signal condition $[F(1,15)=14.94]$. As indicated by Figure 2,

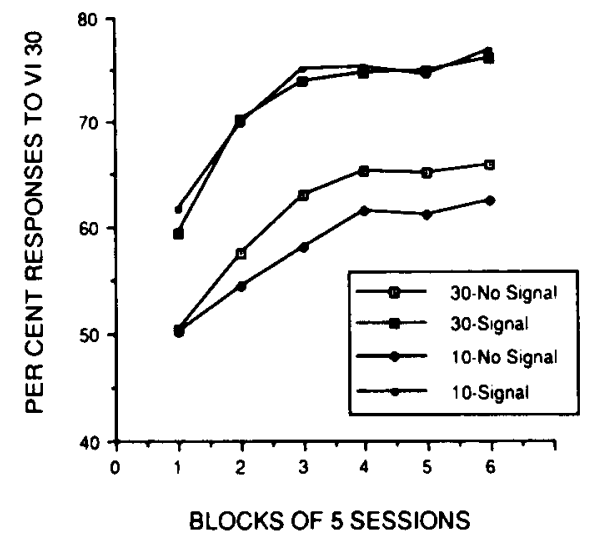

Figure 2. Percentage of the total responses in the two following components (VI $30 \mathrm{sec}$ and VI $6 \mathrm{~min}$ ) that occurred to the higher valued schedule.
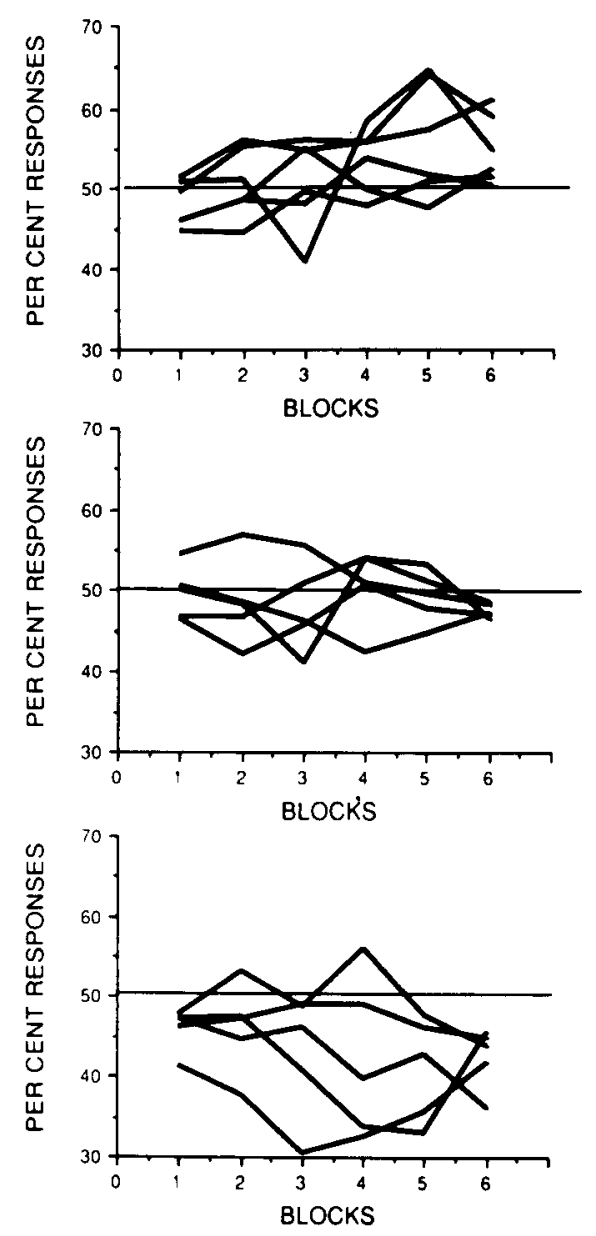

Figure 3. Data for individual subjects from the condition in which the target components were $\mathbf{1 0} \mathrm{sec}$ in duration and no differential signals occurred in the following components (10 sec-No Signal). Shown are the percentages of the total responses during the two target components that occurred during the target followed by the VI 6-min schedule. Values greater than $50 \%$ reflect enhancement of rate by a lower valued following schedule; values less than $50 \%$ reflect a suppression of rate in the tangets by a lower valued following schedule. Each function is the average of the results for the initial and reversal phases of the condition, in order to cancel any effect of stimulus bias.

this was because the 30-sec target components produced greater discrimination than did the 10 -sec targets.

Figure 1 shows that there was no effect of the following schedule when the target components were relatively short and no differential signals occurred in the following components, and that this nondifferential performance remained the same across the $\mathbf{3 0}$ sessions of training. In fact, the similarity in performance in the two targets shown for the 10-sec no-signal condition in Figure 1 hides a great variability among individual subjects. Figure 3 shows the results for individual subjects, subdivided into three categories according to how their performance changed over blocks of training. The ordinate is the percentage of total responses in both target components that occurred to the target followed by the VI 6-min schedule. Hence, values greater than $50 \%$ indicate anticipatory- 
contrast effects; those smaller than $50 \%$ indicate Pavlovian signaling effects. The values shown indicate the average of both the initial and the reversal phases of the condition. Thus, any stimulus-preference effects should have been cancelled between the two phases (see Table 1).

The top group of subjects are those for which the contrast effect increased over sessions. For some of these subjects, this effect was substantial and was similar to that occurring under the other three conditions in which the main effect of the following schedule was statistically significant. The middle group of subjects are those for which there were no differential changes across sessions. The bottom group are the subjects for which the Pavlovian signaling effect increased over sessions. For all groups, the results were highly variable across blocks, with frequent nonmonotonicities in the functions.

Given the relatively poor discrimination of the schedules during the two following components for subjects trained in the 10-sec no-signal condition, seen in Figure 2, the subjects with a high degree of discrimination of the schedule value in the following component might also be those that showed the contrast-type effect (i.e., those shown in the top panel of Figure 3). To test this hypothesis, the relative response rates in the target components during the last 15 sessions of training were correlated with the discrimination performance, also averaged over the last 15 sessions of training. Contrary to this hypothesis, the correlation coefficient (Pearson's rho) was slightly negative $(\varrho=-.16)$. Hence, differential discrimination performance in the following schedule was not a predictor of the pattern of behavior in the target components.

\section{DISCUSSION}

The present results with differential signals in the following components are similar to those from previous free-operant studies of anticipatory contrast (Williams, 1976, 1979, 1981; Williams \& Wixted, 1986; Wilton \& Gay, 1969), in that lower response rates occurred during the target component followed by a higher valued schedule. This effect was enhanced when the target components were relatively shorter, which also replicates previous results (Williams, 1989, Experiment 2). This effect of component duration is not surprising, assuming that anticipatory contrast involves some type of comparison of the reinforcement conditions during the targets with those of the following schedules. With shorter target components, the entire duration of the targets is temporally proximal to the following schedules, so, presumably, the impact of the following schedule should be evident throughout the entire duration of the targets. But with longer target components, the early portions of the targets are still temporally distant from the following schedules, so that the following schedule might be expected to have a reduced influence. Previous studies reporting local response rates within the target components have supported this interpretation, as contrast effects have been greatest at the point of the targets just prior to the transi- tion to the following schedule (Williams, 1988, Experiment 2).

The results when the different following schedules were paired with the same stimulus were more complex. When the target components were relatively long, anticipatory contrast did occur, although it took longer to develop. This presumably is due to the more slowly developing discrimination of the different reinforcement schedules in the following components in comparison to when differential signals were involved. Because the particular target cue was always followed by the same schedule, this allowed differential behavior to occur in the following component to the extent the target cues could be remembered. However, this conditional discrimination was clearly more difficult than the discrimination based on keylight stimuli that were temporally contiguous with the different following schedules, which occurred during the differential signal conditions. Nevertheless, as the conditional discrimination emerged, anticipatory contrast did as well. The occurrence of contrast in the no-signal conditions is contrary to that reported by Williams (1979, Experiments 2 and 3). In that study, subjects initially trained with differential signals in the following components were switched abruptly to the no-signal condition, and anticipatory contrast quickly disappeared during the next two to four sessions, and was replaced by the opposite effect, such that higher response rates occurred in the target component leading to the richer following schedule. Although the procedure used in that study differed from the present procedure in several aspects (e.g., absolute value of component duration, order of presentation of the different conditions), the most likely account of the discrepancy is that training continued only for eight sessions, during which time differential performance in the following schedules did not develop. Thus, those results are directly comparable to the first eight sessions shown in Figure 1, during which anticipatory contrast also did not occur. However, a discrepancy still remains, since the previous study reported a significant effect in the opposite direction, whereas the present results simply show no differential effect.

The results when the no-signal condition was combined with short target components are the most difficult to interpret, and the most important for understanding how anticipatory contrast is related to the embedded Pavlovian contingencies. As seen in Figure 1, no significant difference in response rate occurred in the two target components. But as seen in Figure 3, this is somewhat misleading, since some subjects did show anticipatory contrast while others showed an effect in the opposite direction. This pattern suggests that the processes underlying anticipatory contrast and those underlying autoshaping are separate, and in competition. The role of Pavlovian factors should be the greatest with the 10-sec no-signal condition, both because the absence of other signals should enhance the predictiveness of the target cues for the different following schedules and because shorter duration CSs have been repeatedly demonstrated to enhance Pavlovian 
conditioning (e.g., Gibbon et al., 1977). It is also the procedure that previous work has shown to produce autoshaped responding to the target cues when they are themselves not associated with their own reinforcement schedules (Brown et al., 1982). Presumably as a result of this enhancement of the Pavlovian contingencies, some subjects showed a relative rate enhancement to the target that led to the higher reinforcement rate (bottom pattern of Figure 3). But others showed the opposite effect of anticipatory contrast. It is not evident what differentiated the reactions shown by the different subjects, since the differential performance in the different following components was not a predictor of the type of outcome. Such a pattern can nevertheless be accounted for if the dynamics of anticipatory contrast are assumed to be present in all of the different conditions, and the effect of the Pavlovian contingencies is then superimposed. If the Pavlovian contingencies are sufficiently strong, the result should be that the anticipatory-contrast effect would be overridden, since by definition both cannot be evident given the single measure of response rate used here. The relative influence of contrast versus the Pavlovian contingencies is presumably variable across subjects, with the result that different subjects produce different outcomes.

Support for this interpretation comes from a similar study briefly reported by Hassin-Herman (1984). She also presented pigeons with a four-component multiple schedule, first using the autoshaping procedure of Brown et al. (1982) in which the duration of the target components (in which no reinforcement was available) was varied from 6 to $30 \mathrm{sec}$. Autoshaped responding to the target component leading to the following component with reinforcement available occurred only when the target components were relatively short. In a second experiment, responsecontingent reinforcement was added during the target components themselves, and their duration was again varied. Although her results are somewhat difficult to interpret because only 2 of 4 subjects responded consistently during the target components, the responding that did occur was primarily at the longer component durations, and for both subjects higher response rates occurred in the target component that preceded extinction, rather than that preceding the higher valued VI schedule. Since the only difference between the two experiments was the presence of food in the target components themselves, the difference in their outcome is presumably due to the subject somehow comparing the rates of reinforcement in the target components with those that are to follow. When no reinforcement occurred in the target component, the autoshaping contingencies dominated; when reinforcement was available, the comparison process underlying behavioral contrast predominated, at least with the longer component durations.

Competition between different types of behavioral processes may also explain the variable outcomes obtained in studies of anticipatory contrast using consummatory rather than free-operant behavior. In a series of studies,
Flaherty and his colleagues (Flaherty \& Checke, 1982; Flaherty \& Grigson, 1988; Flaherty \& Rowan, 1985) have shown that licking of a saccharin solution is suppressed if the opportunity to lick is followed by access to a higher valued sucrose solution. Greater suppression occurs the larger the disparity in value and the smaller the temporal separation between the two solutions. Their anticipatorycontrast results are contrary to the enhancement of licking of the target solution found in studies investigating how Pavlovian contingencies produce enhancement of taste preferences. For example, Holman (1975, Experiment 3) presented rats with either of two flavors in a weak saccharin solution, followed by either access to a strong saccharin solution or nothing. The results were that the flavor followed by the preferred strong saccharin solution was enhanced in value, as subjects drank more of it than the alternative when given a preference test. The results were interpreted as showing that a Pavlovian contingency between an initially neutral and a highly palatable substance can produce flavor preferences.

The present results are relevant to the consummatorycontrast procedures because they challenge the interpretation of anticipatory contrast in those procedures in terms of Pavlovian conditioning. Flaherty and his colleagues (e.g., Flaherty \& Grigson, 1988) have argued that the Pavlovian contingency underlies the contrast effect, via the prior saccharin taste eliciting a representation of the sucrose solution that was to follow. This resulted in a direct comparison of the saccharin with the sucrose, and the saccharin was devalued as a result. Flaherty and Grigson acknowledged that this type of Pavlovian effect was different from that normally assumed, whereby the representation of the sucrose would be expected to enhance, rather than reduce, the licking behavior. The present results, to the extent that the dynamics of anticipatory contrast are similar for free-operant and consummatory response procedures, suggest that an interpretation of anticipatory contrast in terms of Pavlovian conditioning is untenable. The target component and the following component did not differ in the type of reinforcer involved, but only in the rate of its delivery. Thus, any representation of the following component elicited by the target stimulus would have to involve rate rather than quality as the critical dimension. More importantly, when the strength of the Pavlovian contingency between the target stimulus and the following component was varied by the experimental manipulations, the condition with the strongest Pavlovian contingency (10-sec, no-signal) was the one condition in which reliable anticipatory contrast did not occur. Such a pattern of results excludes any interpretation of anticipatory contrast in terms of Pavlovian conditioning, unless it is assumed that low to intermediate degrees of Pavlovian contingency produce a form of the conditioned response opposite to the form of the conditioned response produced when the Pavlovian contingency is very strong. However, direct study of Pavlovian conditioning has not revealed any evidence for such an as- 
sumption. Thus, anticipatory contrast appears to be caused by a separate process. Whether it can be reduced to some other more fundamental mechanism remains a mystery.

A final aspect of the results that deserves note is the effect of target duration on the rate of acquisition of the conditional discrimination for the subjects in the two nosignal conditions. Differential behavior in the two following components was possible if the subject used the target stimulus preceding the different following components as a cue. As shown in Figure 2, this discrimination was acquired, albeit slowly. But as also shown there, the discrimination was acquired more quickly when the target stimulus was $30 \mathrm{sec}$ in duration than when it was $10 \mathrm{sec}$. Given that conditional discrimination necessarily involved using the "memory" of the target stimuli, this effect of target duration appears similar to stimulus-exposure effects obtained with delayed matching-to-sample (e.g., Roberts, 1972; Roberts \& Grant, 1974; Spetch \& Treit, 1986) and other complex learning problems (Williams, $1971 \mathrm{a}, 1971 \mathrm{~b}, 1972)$. What is notable about the present results is the range over which the effect occurred. Previous demonstrations of this effect typically have used much shorter exposure times (e.g., 0 to $8 \mathrm{sec}$ ). The present results demonstrate an effect even when the shortest exposure was relatively long, and thus suggest that stimulus duration affects short-term memory over a much larger range than previously believed.

\section{REFERENCES}

Brown, B. L., Hemmes, N. S., Coleman, D. A., JR., Hassin, A., \& Goldhammer, E. (1982). Specification of the stimulus-reinforcer relation in multiple schedules: Delay and probability of reinforcement. Animal Learning \& Behavior, 10, 365-376.

BROWN, P. L., \& JENKINS, H. M. (1968). Auto-shaping of the pigeon's keypeck. Journal of the Experimental Analysis of Behavior, 11, 1-8.

FlaherTy, C. F., \& CHeCKe, S. (1982). Anticipation of incentive gain. Animal Learning \& Behavior, 10, 177-182.

Flaherty, C. F., \& Grigson, P. S. (1988). From contrast to reinforcement: Role of response contingency in anticipatory contrast. Journal of Experimental Psychology: Animal Behavior Processes, 14, 165-176.

Flaherty, C. F., \& Rowan, G. A. (1985). Anticipatory contrast: Within-subjects analysis. Animal Learning \& Behavior, 13, 2-5.
Gibbon, J., Baldock, M. D., Locurto, C., Gold, L., \& Terrace, H. S. (1977). Trial and intertrial durations in autoshaping. Journal of Experimental Psychology: Animal Behavior Processes, 3, 264-284.

Hassin-Herman, A. D. (1984). The role of temporal factors in multiple schedules. In J. Gibbon \& L. Allan (Eds.), Timing and time perception: Annals of the New York Academy of Sciences (Vol. 423, pp. 597-599). New York: New York Academy of Sciences.

Holman, E. (1975). Immediate and delayed reinforcers for flavor preference in rats. Learning \& Motivation, 6, 91-100.

RoBERTS, W. A. (1972). Short-term memory in the pigeon: Effects of repetition and spacing. Joumal of Experimental Psychology, 94, 74-83.

Roberts, W. A., Grant, D. S. (1974). Short-term memory in the pigeon with presentation times precisely controlled. Learning \& Motivation, $5,393-408$.

SPETCH, M. L., \& TREIT, D. (1986). Does effort play a role in the effect of response requirements on delayed matching to sample? Journal of the Experimental Analysis of Behavior, 45, 19-31.

WiLliams, B. A. (1971a). Color alternation learning in the pigeon under fixed-ratio schedules of reinforcement. Journal of the Experimental Analysis of Behavior, 15, 129-140.

Williams, B. A. (1971b). Fixed ratio schedule of reinforcement as a determinant of successive discrimination reversal learning in the pigeon. Psychonomic Science, 25, 143-144.

Williams, B. A. (1972). Probability learning as a function of momentary reinforcement probability. Journal of the Experimental Analysis of Behavior, 17, 363-368.

Williams, B. A. (1976). Behavioral contrast as a function of the temporal location of reinforcement. Journal of the Experimental Analysis of Behavior, 26, 57-64.

Williams, B. A. (1979). Contrast, component duration, and the following schedule of reinforcement. Journal of Experimental Psychology: Animal Behavior Processes, 5, 379-396.

Williams, B. A. (1981). The following schedule of reinforcment as a fundamental determinant of steady-state contrast in multiple schedules. Journal of the Experimental Analysis of Behavior, 35, 293-310.

Williams, B. A. (1988). The effects of stimulus similarity on different types of behavioral contrast. Animal Learning \& Behavior, 16, 206-216.

Williams, B. A. (1989). Component duration effects in multiple schedules. Animal Learning \& Behavior, 17, 223-233.

Williams, B. A., \& WIXTED, J. T. (1986). An equation for behavioral contrast. Journal of the Experimental Analysis of Behavior, 45, 47-62.

WILTON, R. N., \& GAY, R. A. (1969). Behavioral contrast in one component of a multiple schedule as a function of the reinforcement conditions operating in the following component. Journal of the Experimental Analysis of Behavior, 12, 239-246.

(Manuscript received March 30, 1989; revision accepted for publication June 10,1989 .) 